\title{
ERRATUM
}

\section{Annual community patterns are driven by seasonal switching between closely related marine bacteria}

Christopher S Ward, Cheuk-Man Yung, Katherine M Davis, Sara K Blinebry, Tiffany C Williams, Zackary I Johnson and Dana E Hunt

The ISME Journal (2017) 11, 2637; doi:10.1038/ismej.2017.154

Correction to: The ISME Journal (2017) 11, 1412-1422; doi: 10.1038/ismej.2017.4

In the original online publication of this article the incorrect file was uploaded as supplementary information. The correct file has now been added to the online version of the article.

The publishers would like to apologise for this error. 